\title{
TRADUCTORES: TRAIDORES A SU LENGUA MATERNA
}

\author{
María Luisa Masiá Canuto \\ (Universidad Jaume I, Castellón)
}

\begin{abstract}
RESUMEN
Those of us who are engaged in the teaching of Spanish for translators believe that our main objective is that they become good writers in Spanish. Bearing this in mind, on the one hand we must watch out for and detect their most frequent mistakes in order to include them in our syllabus, and, on the other hand we must be capable of applying the most suitable error correction skills. In our opinion, this task cannot be performed if we do not choose the model which best describes the written composition process.

The article ends up with a proposal of an error correction model which we consider as the most appropiate in order to avoid that translators to-be may betray their mother tongue.
\end{abstract}

Afortunadamente, hace tiempo que la afirmación de que todo traductor traiciona al texto original cuando realiza una traducción ha sido prácticamente desterrada. Sin embargo, el traductor sigue siendo un traidor. Traiciona a su lengua materna cada vez que demuestra su desconocimiento de la norma que la rige.

Los profesores de lengua española co-responsables de la formación de futuros traductores tenemos como objetivo fundamental lograr que estos estudiantes sean buenos redactores en dicha lengua. Para ello, es imprescindible, por un lado, detectar cuáles son sus errores más frecuentes y que éstos constituyan el temario de nuestros programas; por otro lado, debemos aplicar aquellas técnicas de corrección de errores que juzguemos más adecuadas. Esta tarea no puede realizarse, a nuestro juicio, aislada de la elección del modelo que mejor describa el proceso de composición escrita.

\section{Modelos del proceso de composición escrita}

\section{Modelo de etapas:}

RoHMAN Y WLECKE (1964) establecieron tres etapas en el proceso de escritura: preescritura o descubrimiento de ideas; escritura y reescritura. Según este 
modelo las etapas son discretas y sucesivas. Las crílicas a esta propuesta se han centrado, por un lado, en la descripción de la naturaleza del proceso de composición -éste dista mucho de ser lineal-, y, por otro, en la ausencia, en este modelo, de los procesos internos del escritor cuando produce un texto.

\section{Modelos cognitivos:}

Hayes y Flower (1980) sí que estudiarán los procesos que el escritor lleva a cabo durante la tarea de composición. Para ello, utilizaron la técnica de hacer pensar en voz alta a los escritores mientras el proceso tenía lugar. Finalmente, claboraron un modelo que consta de los siguientes componentes: memoria a largo plazo del escritor, contexto de producción y proceso propiamente dicho. Las operaciones que configuran este proceso son: planificación, textualización y revisión. El análisis de las operaciones que llevan a cabo los escritores expertos permitió demostrar, además, que éstos realizan constantes idas y venidas entre los diferentes niveles.

HAYES Y FLOWER distinguen tres subprocesos en la planificación: generación de ideas, organización y establecimiento de objetivos:

1) Generación de ideas: se activa en la memoria a largo plazo aquella información relevante en relación con el tema y los posibles destinatarios.

2) Organización de ideas: se selecciona los materiales generados y se ordenan según el tema, la audiencia y la estructura del discurso.

3) Establecimiento de objetivos: se tiene en cuenta para cllo tanto la intención como al lector.

Según Camps (1994: 72), en el modelo de Bronckart, los procedimientos que se emplean en la textualización se agrupan de la siguiente forma: operaciones de conexión, operaciones de cohesión y operaciones de modalización.

1) Operaciones de conexión: su objetivo es encajar los elementos de las estructuras proposicionales «en elements organitzadors d'estructures interproposicionals» e integrarlos en una estructura global.

2) Operaciones de cohesión: contribuyen al mantenimiento de la continuidad del texto.

3) Operaciones de modalización: «constitueixen una mena d'ancoratge comunicatiu a través del qual s'expressa la relació amb els paràmetres de la interacció social. »

Bereiter y SCardamalia (1983 y 1987) defienden el modelo de revisión CDO (comparar, diagnosticar y operar): cuando el mecanismo de comparar detecta algún desajuste, se inicia la operación de diagnosticar, es decir, buscar en el texto y en los conocimientos retóricos de la memoria a largo plazo las causas de ese desajuste. Tras el diagnóstico, se puede operar de dos formas, bien no realizando ninguna corrección o bien llevavando a cabo algún cambio. El proceso se reiniciará tantas veces como sea necesario. 
Este modelo del proceso de composición escrita que hemos desarrollado es el que consideramos más adecuado descriptiva y explicativamente.

\section{Detección de errores}

Chastain (1980:210) considera que el profesor, a la hora de elaborar un inventario de los errores que cometen los estudiantes en sus composiciones, debe tener en cuenta la opinión de los hablantes nativos:

WIIICH STUDIENT LANGUACIL HRRORS are conceived of by instructors as being the most troublesome in their classes? [...]

Which student language errors interfere to the greatest degree with comprehension by native speakers of the language?

Esta propuesta no es válida para nosotros. Y no porque estemos hablando de la enseñanza de una primera lengua. Que lo escrito por nuestros estudiantes sea comprensible para cualquicr hablante nativo puede ser un criterio de corrección adecuado, si lo que buscamos es contribuir simplemente al proceso comunicativo. Sin embargo, los escritos de nuestros estudiantes deben ser además aceptables desde un punto de vista normativo, si lo que queremos es formar a futuros traductores.

Por tanto, pensamos que en este caso la experiencia del profesor que ha trabajado esta habilidad con los estudiantes de traducción puede aportar una información más valiosa. Veamos qué errores suele cometer un estudiante de traducción cuando inicia la Licenciatura:

1) Problemas ortográficos: acentuación y puntuación.

1.1. Ortografia:

1.1.1. Diferenciación de las formas:

porque/porqué/pór qué; adonde/a donde/adónde; a ver/haber;

conque/con que/conqué; sino/si no; demás/de más; aparte/a par-

te; también/tan bien; tampoco/tan poco; así mismo/ asimis-

mo/a sí mismo; ha/a.

1.1.2. Uso de mayúsculas

1.1.3. Verbos con dificultades ortográficas

1.2, Acentuación:

1.2.1. Diptongos, triptongos e hiatos

1.2.2. Demostrativos

1.2.3. Pronombres, adjetivos y adverbios interrogativos

1.2.4. Formas: solo/sólo; aun/aún.

1.2.5. Palabras admitidas con dos acentuaciones 


\subsection{Puntuación:}

1.3.1. Uso de la coma, punto y coma, punto, dos puntos, guiones, paréntesis y comillas

2) Otros problemas gramaticales:

2.1. Determinantes:

\subsubsection{Distributivo SENDOS}

2.1.2. Determinantes delante de sustantivos femeninos que empiezan por $a$

2.2. Pronombres personales: laísmo, leísmo y loísmo.

2.3. Uso de cardinales, ordinales y fraccionarios.

2.4. Verbo:

2.4.1. Utilización del gerundio.

2.4.2. Uso incorrecto de verbos transitivos e intransitivos

2.5. Preposiciones: uso incorrecto.

2.6. Revisión de sinónimos y antónimos.

2.7. Uso de extranjerismos.

3) Dominio de los mecanismos de coherencia:

3.1. Mecanismos de repetición
3.1.1. Deíxis
3.1.2. Anáfora
3.1.3. Definización
3.1.4. Elipsis
3.1.5. Substitución léxica

3.2. Mecanismos de conexión

3.3. Mecanismos de progresión

3.4. Implícitos

3.5. Polifonía enunciativa, registros y variedades

3.6. Tipología textual

\section{Técnicas de corrección de errores}

Para la elección de las técnicas de corrección de errores idóneas, algunas propuestas procedentes de los estudios sobre la enseñanza de segundas lenguas sí nos pueden resultar de gran utilidad.

VANDERGRIFT (1986: 660-662) distingue tres escuelas de pensamiento que presentan tres modelos de corrección de errores.

La primera estaría representada por aquellos que defienden la propuesta de KRASHEN sobre la adquisición de lenguas. Ellos no hacen un gran hincapié en la corrección de los errores en las composiciones. Piensan que si los estudiantes 
son expuestos a modelos correctos de lenguaje escrito, podrán así asimilar las estructuras correctas.

Lamentablemente, a nuestro juicio, aunque consideramos que la exposición a modelos correctos puede ser muy beneficiosa para el estudiante, ésta debe ser complementada con algún tipo de corrección de errores.

La segunda es partidaria de la correccción de errores, pero de una corrección selectiva. Se corregirán sólo aquellos errores que puedan interferir realmente en la comunicación entre los estudiantes y los hablantes nativos.

Esta segunda propuesta no es adecuada para nuestros estudiantes por dos razones fundamentales. La primera ya ha sido mencionada cuando salíamos al paso de las palabras de Chastain, uno de los defensores de esta corrección. Nuestro objetivo no es conseguir que los escritos de nuestros estudiantes sean meramente comprensibles sino que además deben ajustarse a la normativa vigente. La segunda razón nos acerca a la tercera propuesta. En ella se defiende una corrección total de los errores que evite cualquier posible fosilización de los mismos.

La tercera propuesta tiene como principal exponente a LALANDE (1984: 109110) quien revisó la eficacia de ciertas técnicas de corrección de errores gramaticales y ortográficos en las composiciones escritas por sesenta estudiantes de alemán. Tras este estudio, concluye que todas pueden llegar a ser adecuadas desde un punto de vista pedagógico. Veámoslo:

\section{Comprehensive Error Correction}

While selective correction of errors is certainly defensible in the development of speaking skills, the same cannot be said where writing skills are concerned. Unless all errors are identified, the faulty linguistic structures, rather than the correct ones, may become ingrained in the student's interlanguage system. [...]

\section{Systematic Marking of Compositions}

[...] Cohen and Robins [...] indicate that the correction os student compositions is often ineffective in reducing errors because teachers correct mistakes inconsistently. ${ }^{1}$

Guided-Learning and Problem-Solving

Recent literature suggests that the foreign language writing abilities of students could be favorably enhanced through strategies which promote guided-learning techniques. Corder states that simple provision of the correct form may not always be the only, or indeed the most effective, form of correction: «Making a learner try to discover the right form could be more often instructive to both learner and teacher. [... ${ }^{2}$

Vid. Andrew D. Cohen \& Margaret Robbins, «Toward Assessing Interlanguage Perfomance: The Relationship between Selected Errors, Leaners 'Characteristics, and Learners'Explanations», Language Learning, 26, 1976.

$2 \quad$ Vid. S. Pit Corder, «The significance of Learners' Errors», International Review of Applied Linguistics, 5, 1967. 
Instructional Feedback

Put simply, feedback is defined as any procedure used to inform a learner whether an instructional response is right or wrong. [...]

Kulhavy has shown that where instruction in writing is concerned, feedback has its greatest impact on incorrect rather on correct responses. For many years, Skinner and other behaviorists had claimed that feedback had its greatest impact on correct responses. However, Kulhavy has demonstrated that Skinner's position is valid only in regard to spoken language. [...] $]^{3}$

Estas técnicas podrían resumirse así: el profesor debe marcar y corregir todos los errores de forma sistemática - a diferencia de lo que hace cuando trabaja la habilidad oral-, y alternar esta técnica con la de dejar que el estudiante descubra la forma correcta.

\section{Propuesta de nuestro modelo de corrección de errores}

A nuestro juicio, estas técnicas son adecuadas también para corregir los errores orlográlicos y gramaticales de nuestros futuros traductores. Nuestro modelo de corrección tiene en consideración éstas y otras propuestas :

A) Corrección total de los errores de manera sistemática y alternancia de esta técnica con la de marcar simplemente el lugar y tipo de error. Pensamos al igual que WinGFELLD (1974-75: 312) que la autocorrección debe reservarse « [...] for those major errors which we want to remain memorable».

B) Para esta última técnica es imprescindible disponer de un código de corrección de errores. En este código estableceremos una jerarquía de errores similar a la planteada por KENG BoOn (1985: 16-18). Por un lado, distinguimos los crrores globales de los locales. Los primeros afectan a toda la estructura oracional, mientras que los segundos sólo a algún constituyente. Por otro lado, estarán aquellos que afectan al «consumidor»:

1.1. «Harmless»: Error innocuo para la comprensión

1.2. «Confusing»: Error que ocasiona dudas de comprensión.

1.3. «Misleading»: Error que impide la comprensión.

2.1 Lexical.

2.2. Sintáctico.

2.3. Ortográfico.

2.4. Coherencia.

3 Vid.Raymond W. Kulhavy, «Feedback in Written Instruction», Review of Educational Research, 47, 1977. 
3.1. Equivocaciones: Fruto de falta de atención, malos hábitos o deseo de comunicar rápidamente más que correctamente.

3.2. «Mismatches»: Errores lexicales o sintácticos por ignorancia de la forma correcta.

3.3. «Gaps»: Errores debidos a que elementos lexicales o estructuras sintácticas se pierden en lugares donde son obviamente necesarios.

C) Puede ser muy beneficioso para el estudiante elaborar un gráfico que le permita realizar un inventario de los errores cometidos a lo largo del curso. Este inventario puede ser consultado por el estudiante antes de realizar cualquier composición. Esto puede ayudarle a evitar la repetición de los mismos errores de nuevo.

D) La corrección por parte del estudiante de su composición puede ser individual, en parejas o en grupos pequeños. Tanto si es individual como si no lo es, los estudiantes deben recibir ciertas instrucciones que les ayuden. Una recomendación fundamental es que lean varias veces lo escrito, focalizando su atención en aspectos concretos de naturaleza ortográfica, gramatical o de coherencia.

E) La puesta en común del trabajo realizado por un alumno o un grupo de ellos, por medio de un retroproyector, posibilita una nueva corrección del escrito.

F) Se trata, por tanto, de enseñar al estudiante a elaborar varios borradores antes de considerar el escrito como definitivo. Tras ser corregido cada borrador, primero por el estudiante y después por otra persona o personas de la clase, el estudiante puede entregar la última versión al profesor. Éste podrá proponer correcciones o simplemente señalar alguna de ellas. Tras seguir las recomendaciones del profesor, el estudiante elaborará una composición definitiva.

Pero ¿qué sucede con los errores que cometen con los mecanismos de coherencia? ¿Son igualmente válidas estas técnicas de corrección?

G) Para la corrección de errores en el dominio de los mecanismos de coherencia, pensamos que, en principio, las técnicas de corrección son válidas. Sin embargo, para que el estudiante llegue realmente a entender nuestra propuesta de corrección, o si no hay propuesta qué incorrección es la que debe trabajar por su cuenta, es necesario que éste tenga oportunidad de mantener una pequeña charla con el profesor fuera del aula.

Todos somos conscientes de que con clases de setenta estudiantes difícilmente podemos atender en nuestras tutorías todas y cada una de las composiciones que estos estudiantes redactan a lo largo del curso, aunque sí un buen número de ellas. El resto pueden ser corregidas mediante el uso de un casete. HALLET (1983: 30-31) afirma que aunque las grabaciones en un casete no pueden igualar una entrevista personal, es lo más próximo a ella 
Marking the papers is no problem. [...] Problems of content, organization, or vocabulary choice can be numbered, underscored, circled, or otherwise highlighted, perhaps with a brief note to the teacher so he does not forget why he circled it. ${ }^{4}$

\section{Conclusión}

Ésta es nuestra propuesta de los errores de escritura en que debe hacer hincapié el profesor de español como lengua materna para futuros traductores y qué modelo de corrección de los mismos juzgamos más adecuado.

Keng Boon (1985: 20) finaliza su artículo afirmando lo siguiente:

The strategies mentioned will change the role of the teacher in the marking of compositions. From an omniscient authority who hands down the correct usage of the language, he becomes a consultant, a source of guidance and help. [...] It also necessitates a more tolerant attitude towards errors which conforms to the present emphasis on fluency.

Coincidimos plenamente en el papel que debe representar el profesor tal y como lo presenta Keng Boon. Sin embargo, si queremos evitar que nuestros futuros traductores puedan ser tildados de traidores a su lengua, la tolerancia frente al error en pro de mayor fluidez no parece ser el camino más adecuado.

\section{Bibliografía}

Beretter, C. Y M. SCARdamalia (1983): «Does learning to write have to be so difficult?», A. Freedman, I. Pringle y J. Yalden (eds.): Learning to Write: First Language/Second Language, Londres, Longman, pp. 20-33.

Bereter, C. (1987): The Psychology of Written Composition, Hillsdale, N. J., Erlbaum.

Brodkey, D. Y R. Young (1981): «Composition Correctness Scores», TESOL Quarterly, 15, 2, pp. 159-167.

4 Pensamos que puede resultar de interés conocer alguna de las opiniones de estudiantes que han tenido esta experiencia con las cintas magnetofónicas. Veamos las que recoge Farnsworth (1974) de sus estudiantes:

I rather prefer the using of tape cassettes to the comments on my paper because it isn't possible to explain the errors in such a little space; however, if the teacher uses a tape, she can tell us the error and also the way she thinks it could be made better.

I prefer having my composition corrected using the tape method because it is more precisely commented on than the standart use of comments in the margins of my paper.

The cassette gives more information about my papers and also I can understand 
Camps, A. (1994): L'ensenyament de la composició escrita, Barcelona, Barcanova. CARTER, M. E. (1975): Error Analysis in the Free Compositions of Spanish Speakers, Tesis Doctoral de la Universidad de Georgetown.

CoHEn, A. D. y M. Robbins (1976): «Toward Assessing Interlanguage Performance: The Relationship between Selected Errors, Learners 'Characteristics, and Learners' Explanations», Language Learning, 26, 1, pp. 45-66.

Corder, S. P. (1967): «The Significance of Learner's Errors», International Review of Applied Linguistics, 5, pp. 161-170.

Chastain, K. (1980): «Native Speaker Reaction to Instructor-Identified Student Second Language Errors», Modern Language Journal, 64, pp. 210-215.

Chastain (1981): «Native Speaker Evaluation of Student Composition Errors», Modern Language Journal, 65, pp. 288-294.

FARNSWORTH, M. B. (1974): «The cassette tape recorder: A bonus or a bother in ESL composition correction», TESOL Quarterly, 8, 3.

FRIEDEnBERG, J. E. (1979): The Acquisition of Spanish: An examination of Learner Errors and Strategies, Tesis Doctoral de la Universidad de Illinois. Guberman, S. (1988): «Étude comparée de trois approches à la correction des erreurs dans les compositions écrites», The Canadian Modern Language Review, 44, 4, pp. 702-714.

HaLlet, B. (1983): «Correcting ESL Compositions with a Cassette Recorder: Getting to Know the Reader, Nor the Proofreader», TESL Reporter, 16, pp. 29-32.

HAYES, J. R. Y L. Flower (1980): «Identifying the organization of writing process», L. W. Gregg y E. R. Steinberg (eds.): Cognitive Processes in Writing, Hillsdale, N. J., Lawrence Erlbaum, pp. 3-30.

Hendrickson, J. M. (1980): «The Treatment of Error in Written Work», Modern Language Journal, 64, pp. 216-221.

Keng Boon, $\mathrm{CH}_{\mathrm{H}}$ (1985): «Error Analysis and Composition Marking», Guidelines. A Periodical for Classroom Language Teachers, 7, 1, pp. 13-21.

LAlande, J. F. (1984): «Reducing Composition Errors: An Experiment», Foreign Language Annals, 17, 2, pp. 109-117.

LeVEnston, E. A. (1978): «Error analysis of free composition: the theory and the practice», Indian Journal of Applied Linguistics, IV, 1, pp. 1-11.

Rohman, D. G. Y A. O. Wlecke (1964): Pre-writing: The construction and application of models for concept formation in writing, East Lansing, Michigan State University. U. S. Office of Education Cooperative Research Project, núm. 2174.

Rosen, L. M. (1987): «Developing Correctness in Student Writing: Alternatives to the Error-Hunt», English Journal, 76, 3, pp. 62-69.

SCHACHTER, J. (1979): «Reflections on Error Production», Interlanguage Studies Bulletin, 4, 1, pp. 15-26. 
Schachter, J. y M. Celce-Murcia (1977): «Some Reservations Concerning Error Analysis», TESOL Quarterly, 11, 4, pp. 441-451.

VANDERGRIFT, L. (1986): «Second Language Writing and Correction: Toward and Improved Model for Composition Correction», The Canadian Modern Language Review, 42, 3, pp. 658-667.

WiNGFIELD, R. J. (1974-75): «Five Ways of Dealing with Errors in Written Composition», English Language Teaching, 29, pp. 311-313. 